\section{Reply to Mitchell}

\section{SAM REVUSKY Memorial University of Newfoundland}

Mitchell, Scott, and Mitchell (1977) referred to "the near universal omission of the appropriate noncontingency poisoned or sham injected control groups" in long-delay taste aversion experiments. Examples cited were papers by Garcia, Ervin, and Koelling (1966), Revusky (1968), and Smith and Roll (1967). In defense, I pointed out that each of these papers contained both types of controls (Revusky, 1977). In a reply, Mitchell (1977) did not deny that each paper contains sham controls, but insisted that none of them contains adequate noncontingent poisoned controls. According to Mitchell, "when animals poisoned some hours after consuming the CS do not subsequently avoid it," Revusky calls them noncontingent poisoned controls, but "when similarly treated animals avoid the CS, they constitute an experimental group which has formed an association over a long delay (Revusky, 1968)."

I was not so arbitrary. I had pointed to failures by Garcia et al. (1966) and by Smith and Roll (1967) to obtain aversions at their longest delays of poisoning as controls for aversions obtained with shorter delays in those same experiments. These are proper noncontingent poisoning controls because they control for the possibility that the taste aversions are unrelated to the contingency between the taste and the poisoning. When longer delays of sickness do not produce an aversion, the aversions obtained with shorter delays must be due to the contingency of the taste with the sickness. Such controls are excellent because all groups are equated with respect to familiarity with the test solution, history with the comparison solution (if a two-choice test is used), and experience with sickness. Furthermore, in all the experiments questioned by Mitchell, orderly delay gradients supplied further evidence that the aversions depended upon the contingency between the taste and the sickness.

An analysis of the experiment by Mitchell et al. (1977) shows that the controls Mitchell considers adequate are far inferior to the controls he has criticized. Table 1 designates each group in the experiment by whether saccharin solution ("Sac") or unflavored water (" $\mathrm{H}_{2} \mathrm{O}$ ") was consumed during a training trial and by whether immediate sickness ( $25 \mathrm{mg} / \mathrm{kg}$ of cyclophosamide), delayed sickness (by $6 \mathrm{~h}$ ), or placebo injection followed. The second column contains preferences obtained for saccharin solution (relative to unflavored water) on test Day 1 for each group and is based on raw data kindly supplied to me by Mitchell. The last four columns indicate determinants of saccharin preference which must be considered in any study of taste aversion learning; a "yes" means the indicated factor is applicable to the indicated group and a " + " or" -" means its effect is to increase or decrease saccharin preference. Table 1 shows that the two planned experimental groups, Sac $\rightarrow$ ImmedSick and Sac $\rightarrow$ DelaySick, are not properly equated with any of the remaining groups with respect to extraneous determinants of saccharin preference as follows.

Familiarity with saccharin. The groups that drank saccharin solution during training are designated " +" for this factor because familiarity with a flavor increases the preference for it (Revusky \& Garcia, 1970). This is confirmed by the higher saccharin preference of Group Sac $\rightarrow$ Placebo than Group $\mathrm{H}_{2} \mathrm{O} \rightarrow$ Placebo $^{1}: t(20)=6.56, p<.001$. Mitchell's planned noncontingent poisoning control groups, $\mathrm{H}_{2} \mathrm{O} \rightarrow$ ImmedSick and $\mathrm{H}_{2} \mathrm{O} \rightarrow$ DelaySick, are not equated with his planned experimental groups, Sac $\rightarrow$ ImmedSick and Sac $\rightarrow$ DelaySick, on this factor.

Learned $\mathrm{H}_{2} \mathrm{O}$ aversion. Rats readly learn aversions to unflavored water (Elkins, 1974; Garcia \& Koelling, 1967; Revusky \& Parker, 1976). Group $\mathrm{H}_{2} \mathrm{O} \rightarrow \mathrm{Im}$ medSick is designated " + "for this factor because such an aversion was likely to increase its relative preference for saccharin solution during the twochoice test in which the alternative was unflavored water; this is marginally confirmed by its higher preference for saccharin than $\mathrm{Group} \mathrm{H}_{2} \mathrm{O} \rightarrow$ DelaySick, which was equated with it on everything but delay of sickness: $\mathrm{t}(20)=2.08, .05<\mathrm{p}<.06$, two tails. Failure to control for an aversion to unflavored

Table 1

Saccharin Preferences Obtained by Mitchell, Scott, and Mitchell (1977) and an Analysis of their Determinants

\begin{tabular}{lccccc}
\multicolumn{1}{c}{ Group } & $\begin{array}{c}\text { Day 1 } \\
\text { Preference }\end{array}$ & $\begin{array}{c}\text { Familiar } \\
\text { With Sac }\end{array}$ & $\begin{array}{c}\text { Learned } \mathrm{H}_{2} \mathrm{O} \\
\text { Aversion }\end{array}$ & $\begin{array}{c}\text { Sick During. } \\
\text { Test }\end{array}$ & $\begin{array}{c}\text { Leasned Sac } \\
\text { Aversion }\end{array}$ \\
\hline Sac $\rightarrow$ ImmedSick & .07 & Yes (+) & & Yes $(-)$ & Yes $(-)$ \\
$\mathrm{H}_{2} \mathrm{O} \rightarrow$ DelaySick & .13 & & & Yes (-) \\
$\mathrm{H}_{2} \mathrm{O} \rightarrow$ Placebo & .23 & & Yes (+) & Yes (-) \\
$\mathrm{H}_{2} \mathrm{O} \rightarrow$ ImmedSick & .28 & & & Yes (-) \\
$\mathrm{Sac} \rightarrow$ DelaySick & .36 & Yes (+) & & \\
Sac $\rightarrow$ Placebo & .62 & Yes (+) & & & \\
\hline
\end{tabular}


water was a more serious shortcoming in Mitchell's experiment than in other experiments because Mitchell tested on the day after training so that there was no chance for the aversion to extinguish during recovery sessions. For instance, in cyclophosamide experiments a three-day recovery period has been standard.

Sickness during testing. This was a likely determinant of the results because Group $\mathrm{H}_{2} \mathrm{O} \rightarrow$ DelaySick had a lower saccharin preference than Group $\mathrm{H}_{2} \mathrm{O} \rightarrow$ Placebo, $\mathrm{t}(20)=2.22, \mathrm{p}<.05$; thus all rats injected with cyclophosamide were assigned "_-" for this factor in Table 1. Those specifically criticized by Mitchell (1977) for lack of appropriate noncontingent poisoning controls carefully showed that sickness during testing did not affect their results. Mitchell's (1977) belated attempt to show the same was based on a comparison between Groups $\mathrm{H}_{2} \mathrm{O} \rightarrow$ ImmedSick and $\mathrm{H}_{2} \mathrm{O} \rightarrow$ Placebo; Table 1 shows this to be invalid. Sickness during testing was an important factor because Mitchell et al. (1977) did not allow enough time for recovery from sickness.

Learned saccharin aversions. Table 1 shows that none of the original control groups can validly be used to determine if saccharin aversions in Groups Sac $\rightarrow$ ImmedSick and Sac $\rightarrow$ DelaySick are due to learning. However, since $t(20)=3.88$, $\mathrm{p}<.001$, between these two groups, it can be inferred that Group Sac $\rightarrow$ ImmedSick exhibited a learned aversion provided Mitchell's (1977) own criteria for adequate controls are ignored. Group Sac $\rightarrow$ DelaySick had a lower saccharin preference than Group Sac $\rightarrow$ Placebo, $t(20)=2.98$, $\mathrm{p}<.01$, but Table 1 shows this can either be due to associative learning or due to sickness during the test. I incline to the latter explanation by extrapolating from results by Dragoin (1971) and Wright, Foshee, and McCleary (1971). I do not know why Mitchell et al. expected a learned aversion at a 6-h delay with a $25 \mathrm{mg} / \mathrm{kg}$ dose of cyclophosamide.

Defense of Revusky (1968). In response to my criticism of his failure to deal with sickness during the test, Mitchell (1977) counterattacked: "although he readily accepts his own data from 'sick' animals, he questions ours." In reality I used explicit irradiated control groups to prove that there was no effect of sickness on preference tests administered 3-5 days after irradiation (Revusky, 1968). Later in his critique, Mitchell criticized these irradiated controls because all groups had been made equally familiar with the sugar test solution and because all groups were given identical experience with the milk solution which was to be an alternative to the sugar solution in the two-bottle test. In fact, it was by these very features that I avoided the methodological chaos into which Mitchell et al. fell. Mitchell also objected that I used only two rats per control group; in reality, the two rats were a subgroup used in a secondary statistical test to show that the results were significant even under the least sensitive conditions. Finally, there is no need to discuss hypophagia produced by irradiation and its implications because Revusky (1968) used proper controls; for my opinion about this, see Revusky and Garcia (1970, p. 50).

\section{REFERENCES}

Dragous, W. B. Conditioning and extinction of taste aversion with variations in intensity of the CS and US in two strains of rats. Psychonomic Science, 1971, 22, 303-305.

Exuss, R. L. Conditioned flavor aversions to familiar tap water in rats: An adjustment with implications for aversion therapy treatment of alcoholism and obesity. Joumal of Abnormal Psychology, 1974, 83, 411-417.

Garcia, J., Ervin, F. R., \& Kozlling, R. A. Learning with prolonged delay of reinforcement. Psychonomic Science, 1966, 5, 121-122.

Garcia, J., \& Koelling, R. A. A comparison of aversions induced by $\mathrm{X}$-rays, toxins, and drugs in the rat. Radiation Research Supplement, 1967, 7, 439-450.

Mrtchell, D. Reply to Revusky. Animal Learning \& Behavior, 1977, 5, 321-322.

Mrtchell, D., Scotr, D. W., \& Mrtchell, L. K. Attenuated and enhanced neophobia in the taste-aversion "delay of reinforcement" effect. Animal Learning \& Behavior, 1977, 5, 99-102.

Revusky, S. H. Aversion to sucrose produced by contingent X-irradiation: Temporal and dosage parameters. Journal of Comparative and Physiological Psychology, 1968, 65, 17-22.

Revusxy, S. Correction of a paper by Mitchell, Scott, and Mitchell. Animal Learning and Behavior, 1977, 5, 320.

Revusry, S., \& Garcis, J. Learned associations over long delays. In G. H. Bower (Ed.), The psychology of learning and motivation: Advances in research and theory (Vol. 4). New York: Academic Press, 1970.

Revusky, S., \& Parker, L. A. Aversions to unflavored water and cup drinking produced by delay of sickness. Journal of Experimental Psychology: Animal Behavior Processes, 1976, 2, 343-353.

SyItH, J. C., \& Roll, D. L. Trace conditioning with X-rays as the aversive stimulus. Psychonomic Science, 1967, 9, 11-12.

Wriger, W. E., Fosher, D. P., \& McCleary, G. E. Comparison of taste aversions with various delay and cyclophosphamide dose levels. Psychonomic Science, 1971, 22, 55-56.

\section{NOTE}

1. Ordinary two-group $t$ tests are used here instead of the Newman-Keuls statistics supplied by Mitchell et al. (1977) for two reasons. (1) The factors delineated in Table 1 have been known for over a decade, and our use of statistics is simply to confirm that they were present in Mitchell's experiment. The Newman-Keuls test is appropriate for blind statistical situations, but not for assessment of whether known effects were replicated. (2) There was pronounced inhomogeneity in the data $\left(\mathrm{F}_{\max }=\right.$ 58.9 , where $F_{\max }=10.4$ has $p=.01$ ). Since the Newman-Keuls test is based on a single experimentwise estimate of error, it obscures differences where both groups have variances substantially smaller than is average for the experiment. The practical implication of our failure to use a pooled estimate of variance is that we will report an effect significant at $p<.05$, two tails, for a reduction in saccharin preference as a result of sickness during testing; had pooled variance been used, $t(60)=1.42, p<.10$, one tail.

(Received for publication May 27, 1977; revision accepted October 20,1977 .) 